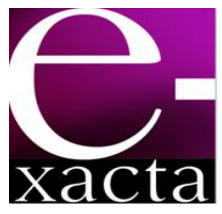

ISSN: 1984-3151

\title{
O FATOR DE POTÊNCIA EM UNIDADES CONSUMIDORAS RESIDENCIAIS
}

\author{
THE Power Factor in Residential Consumer Units
}

\section{Frederico Santiago Romualdo Rios ${ }^{1}$; Gustavo Sacchetto Curi2; Fabricio Silveira Chaves; Arlete Vieira da Silva ${ }^{4}$}

1 Engenheiro Eletricista. UNIBH, 2013. Eletromecap Industrial Ltda. Belo Horizonte, MG. fredsantiago@hotmail.com

2 Engenheiro Eletricista. UNIBH, 2013. Projen Projetos em Engenharia Ltda. Belo Horizonte, MG. gustavoscuri@gmail.com

3 Doutor em Engenharia Elétrica. UFMG, 2012. Professor do Centro Universitário de Belo Horizonte UNI-BH. Belo Horizonte, MG. fabricio.chaves@prof.unibh.br

4 Mestre em Geografia e Análise Ambiental. UFMG, 2002. Belo Horizonte, MG. S \& V Consultoria e Serviços Ltda. svconsultoriaeservicos@gmail.com.

Recebido em: 06/09/2013 - Aprovado em: 20/04/2014 - Disponibilizado em: 31/05/2014

RESUMO: Este trabalho apresenta um estudo das vantagens da correção local da energia reativa nas unidades consumidoras residenciais, buscando otimizar os reativos circulantes na instalação local e na rede de distribuição secundária, visto que a carga de uma instalação residencial tem baixo fator de potência. Tal situação acarreta elevadas perdas na rede de distribuição das concessionárias e nas instalações elétricas residenciais, que normalmente são subdimensionadas e exigidas ao máximo devido ao grande número de equipamentos eletroeletrônicos utilizados atualmente. A compensação dos reativos pode evitar a tarifação destes em uma eventual cobrança por parte das concessionárias. Neste trabalho é feito um breve estudo sobre a natureza da energia reativa e das cargas residenciais, bem como um estudo de caso, no qual foram analisadas as cargas mais comuns em uma residência. Em seguida, foram apresentados os procedimentos de cálculo e dimensionamento do compensador (capacitor), visando demonstrar as vantagens da correção de reativo residencial, avaliando tanto a sua viabilidade econômica quanto os impactos na redução das perdas na rede de distribuição. Considerando que, no caso estudado, com a correção do fator de potência foi obtida uma redução de 9,85\% da corrente, utilizando um controlador automático e capacitores de $20 \mathrm{uF}, 10 \mathrm{uF}$ e $5 \mathrm{uF}$. Dentre várias vantagens, esta redução de corrente circulante no sistema permitirá uma menor utilização das usinas geradoras e uma redução nas perdas no sistema de transmissão e distribuição. Esses são dois entre vários fatores que justificam a correção do fator de potência para as residências, beneficiando de forma significativa o sistema de geração e transmissão de energia.

PalavRAS - Chave: Correção Fator de Potência. Energia Reativa. Perdas Elétricas. Tarifação de Reativos.

ABSTRACT: This paper presents a study of the advantages of local correction of reactive power in the residential consumer units, seeking to optimize the reactive circulating in the local installation and the secondary distribution network, since the load of a residential facility has a low power factor, this leads to high losses in the distribution network of dealers and residential electrical installations which are usually undersized and required the most due to the large number of electronic equipment used today. The compensation of reactive can avoid these charges in an eventual recovery by concessionaires. It made a brief study of the nature of the reactive power and residential loads, as well as a case study which analyzes the most common charges in a residence. Then presents the procedures for calculation and dimensioning the compensator (capacitor), the aim is to demonstrate the advantages of correction reactive residential, evaluating both its economic viability as the impacts in reducing 
losses in the distribution network. Whereas, in the case studied, correcting the power factor was obtained a reduction of $9.85 \%$ of the current, using an automatic controller and $20 \mathrm{uF}, 10 \mathrm{uF}$ and $5 \mathrm{uF}$ capacitors. Among various advantages, this reduction in system circulating current allows a smaller use of power plants and a reduction of system losses in transmission and distribution. These were two of many factors that justify the correction of power factor for residential, benefiting significantly the system of generation and transmission of energy.

KEYWORDS: Correction, reactive power, power losses, power factor, reactive power pricing.

\section{INTRODUÇÃO}

O crescente aumento na demanda do sistema elétrico, aliado à escassez de recursos de geração, tem aumentado a busca de novas tecnologias para melhorar o aproveitamento dos recursos existentes com a constante preocupação de se minimizar os investimentos.

Para os consumidores de pequeno porte, do tipo residencial, ainda não existem contratos do tipo horosazonal, mas os novos medidores instalados pelas concessionárias de energia já têm a capacidade de mensurar o fator de potência reativo.

Diante dos fatos ocorridos no ano de 2001, em relação ao racionamento de energia elétrica e à deficiência do Sistema Interligado Nacional (SIN) na capacidade de transmissão e geração, as concessionárias de energia podem implementar a cobrança de potência reativa nas residências.

O trabalho pretendeu analisar os valores de potência reativa em residências e diminuir esses valores para que melhore ainda mais a qualidade energia fornecida no país.

A correção do fator de potência é um assunto bastante discutido devido a sua grande importância no consumo de energia elétrica. De acordo com Creder (2007), um baixo fator de potência pode causar sobrecarga em cabos e transformadores, gerando um aumento nas perdas do sistema, nas quedas de tensão e no desgaste em dispositivos de proteção e manobra.
O mesmo autor sugere um método mais difundido para correção do fator de potência, que consiste na instalação de bancos de capacitores em paralelo à rede elétrica, devido ao seu menor custo de implantação e por serem equipamentos estáticos de baixo custo de manutenção.

A pesquisa sobre a correção do fator de potência é necessária para verificar a viabilidade da correção, pois as concessionárias de energia elétrica já cobram um ajuste financeiro $(R \$)$ sobre o fator de potência, quando este é inferior a 0,92 para os consumidores do Grupo A, atendidos em média e alta tensões (CEMIG, 2003).

\section{RefERENCIAL TEÓRICO}

A energia elétrica é a forma de energia que pode ser transportada com maior facilidade. Para chegar às residências, ruas, comércio e indústrias, ela percorre um longo caminho a partir das usinas geradoras.

A energia elétrica passa por 3 etapas principais, 0 que, segundo a Companhia Energética de Minas Gerais (CEMIG, 2003), consiste em:

a) Geração: A energia elétrica é convertida a partir da energia mecânica de uma turbina que movimenta um gerador. Essa energia mecânica é gerada por diferentes fontes primárias, como a força da água que cai (hidráulica), o vapor (térmica), que pode ter origem na queima do carvão, óleo combustível, gás natural, ou ainda a energia nuclear. 
b) Transmissão: As usinas geradoras normalmente se situam distantes dos centros consumidores de energia elétrica. Por isso é preciso transportar a energia elétrica produzida nas usinas até os locais de consumo: cidades, indústrias, propriedades rurais, dentre outros. Para isso, são construídas as Subestações Elevadoras de Tensão e as Linhas de Transmissão.

c) Distribuição: Nas cidades estão construídas as subestações transformadoras. Sua função é baixar a tensão do nível de Transmissão (muito alto) para o nível de Distribuição.

Estas etapas podem ser representadas conforme apresentado na Figura 1.

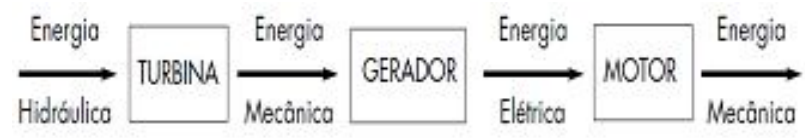

Figura 1 - Etapas de Geração, Transmissão, Distribuição e Utilização.

Fonte: CEMIG, 2003, p. 19

\subsection{POTÊnCIA ElÉtRICA}

As Unidades de Medidas do Sistema Internacional encontram-se na Tabela 1.

Tabela 1 - Unidades de Medidas do Sistema Internacional

\begin{tabular}{|c|c|c|}
\hline \multicolumn{3}{|c|}{ UNIDADES ELÉTRICAS } \\
\hline UNIDADE & SÍMBOLO & DETERMINA \\
\hline Ampere & $\mathrm{A}$ & Corrente Elétrica \\
\hline Volt & $\mathrm{V}$ & Tensão Elétrica \\
\hline Watt & $\mathrm{W}$ & $\begin{array}{c}\text { Potência Elétrica } \\
\text { Ativa }\end{array}$ \\
\hline Volt-Ampere & $\mathrm{VA}$ & $\begin{array}{c}\text { Potência Elétrica } \\
\text { Aparente }\end{array}$ \\
\hline $\begin{array}{c}\text { Volt-Ampere } \\
\text { reativo }\end{array}$ & var & $\begin{array}{c}\text { Potência Elétrica } \\
\text { Reativa }\end{array}$ \\
\hline Watt-hora & Wh & $\begin{array}{c}\text { Potência Elétrica } \\
\text { Ativa por hora }\end{array}$ \\
\hline
\end{tabular}

Fonte: CEMIG, 2003, p. 16, adaptado.
Conforme CEMIG (2003), a Potência Elétrica (P) é definida como o trabalho efetuado na unidade do tempo. Essa potência é calculada através da multiplicação da Tensão pela Corrente Elétrica que passa por um circuito. A unidade da Potência Elétrica é o watt e o seu símbolo é o W.

\subsection{ResoluÇÃo ANEel № 414/2010}

A Agência Nacional de Energia Elétrica - ANEEL - é uma autarquia vinculada ao Ministério de Minas e Energia, que foi criada há quase 18 anos pela Lei 9.427, quando ocorreu a publicação no Diário Oficial da União em 26/12/1996 (ANEEL, 2000).

O mesmo autor da resolução ressalta que suas atribuições consistem em regular e fiscalizar a geração de energia elétrica no país, bem como a sua distribuição e comercialização, levando em consideração a defesa do interesse do consumidor.

Também é sua atribuição mediar os conflitos de interesse que possam ocorrer entre os agentes do setor elétrico, bem como aqueles que venham a surgir entre os mesmos agentes e os consumidores (ANEEL, 2010).

O redator afirma também ser responsável por conceder, permitir ou autorizar a instalação e serviços de energia, cuidando da prática das tarifas justas e pelo zelo na qualidade do serviço. Além de exigir investimentos, promovendo também estímulos à competição entre as operadoras, assegurando que os serviços prestados sejam de abrangência universal, ou seja, que sirvam a todos os consumidores.

Entretanto cabe ressaltar que a ANEEL vem trabalhando para proporcionar condições favoráveis para que o mercado de energia elétrica se desenvolva com equilíbrio entre os agentes e em benefício da sociedade. 
A Resolução ANEEL № 414/2010, em substituição à Resolução ANEEL № 456/2000, estabelece, de forma atualizada e consolidada, as Condições Gerais de Fornecimento de Energia Elétrica e descreve em seus Art. 95 e 97 que:

Art. 95. O fator de potência de referência "fR", indutivo ou capacitivo, tem como limite mínimo permitido, para as unidades consumidoras dos grupos A e B, o valor de 0,92.

Parágrafo único. Aos montantes de energia elétrica e demanda de potência reativos que excederem o limite permitido aplicam-se as cobranças estabelecidas nos arts. 96 e 97, a serem adicionadas ao faturamento regular.

Art. 97. Para unidade consumidora que não possua equipamento de medição que permita a aplicação das equações fixadas no art. 96, os valores correspondentes à energia elétrica e demanda de potência reativas excedentes são apurados conforme as seguintes equações:

Ere $=E E A M \times\left(\frac{f r}{f m}-1\right) \times$ VRere

Dre $=\left(P A M \times \frac{f r}{f m}-P A F\right) \times$ VRdre

Ere = valor correspondente à energia elétrica reativa excedente à quantidade permitida pelo fator de potência de referência, no período de faturamento, em Reais $(R \$)$;

EEAM = montante de energia elétrica ativa medida durante o período de faturamento, em megawatt-hora (MWh);

$\mathrm{fr}=$ fator de potência de referência igual a 0,92;

$\mathrm{fm}=$ fator de potência indutivo médio da unidade consumidora, calculado para o período de faturamento;

VRere = valor de referência equivalente à tarifa de energia "TE" aplicável ao subgrupo B1, em Reais por megawatt-hora $(\mathrm{R} \$ / \mathrm{MWh})$;

Dre $=$ valor correspondente à demanda de potência reativa excedente à quantidade permitida pelo fator de potência de referência, no período de faturamento, em Reais $(R \$)$;
$\mathrm{PAM}=$ demanda de potência ativa medida durante $\mathrm{O}$ período de faturamento, em quilowatt $(\mathrm{kW})$;

PAF = demanda de potência ativa faturável no período de faturamento, em quilowatt $(\mathrm{kW})$;

VRdre = valor de referência, em Reais por quilowatt $(R \$ / k W)$, equivalente às tarifas de demanda de potência para o posto tarifário fora de ponta - das tarifas de fornecimento aplicáveis aos subgrupos do grupo A para a modalidade tarifária horária azul. (ANEEL, 2010, p. 61).

No artigo 95, pode-se perceber que a cobrança da potência reativa também poderá ser realizada para consumidores classe "B". O artigo 97 estabelece os procedimentos de cálculo para apuração da demanda de reativos para consumidores que não possuem o equipamento específico para medi-lo, como os consumidores da classe B1(residenciais).

\subsection{FATOR DE POTÊNCIA}

De acordo com CEMIG (2003), em um condutor elétrico energizado em Corrente Alternada (CA), passa uma determinada quantidade de energia, sendo um percentual Ativo e outro Reativo. Quanto maior for o percentual de Potência Ativa (kW) que passar, este será melhor e mais econômico.

O mesmo autor afirma que a Potência Reativa (kvar) é necessária para criar o fluxo magnético necessário para o funcionamento de equipamentos como motores, transformadores, dentre outros, mas esta potência não realiza trabalho. A Potência Ativa (kW) é a que efetivamente produz trabalho.

Conforme o mesmo autor, para que se tenha uma ideia da diferença entre potência ativa e reativa, é dado um exemplo de uma forma bastante simplificada, fazendo uma analogia com um copo cheio de cerveja.

Num copo cheio de cerveja, há uma parte ocupada pelo líquido e outra ocupada pela espuma. Para aumentar a quantidade de líquido nesse copo, tem-se que diminuir a espuma. Assim, de maneira 
semelhante ao copo com cerveja, a Potência Elétrica solicitada, por exemplo, por um motor elétrico, é composta de Potência Ativa (kW), que "corresponde" ao líquido, e Potência Reativa (kvar), que "corresponde" à espuma (CEMIG, 2003).

Como se pode verificar na Figura 2, a soma vetorial das Potências Ativa e Reativa é denominada de Potência Complexa (kVA) e seu módulo é a Potência Aparente (kVA), representado pelo vetor $S$ do triângulo. Como a rede tem um limite de capacidade de transmissão, a capacidade em kVA de um circuito elétrico (fiação, transformadores, dentre outros) é limitada também. Para aumentar a Potência Ativa (representada por $\mathrm{P}$ no triângulo) em um circuito, é preciso reduzir a Potência Reativa Q. O Fator de Potência (FP) é definido como o cosseno do ângulo entre as retas $S$ e $P$.

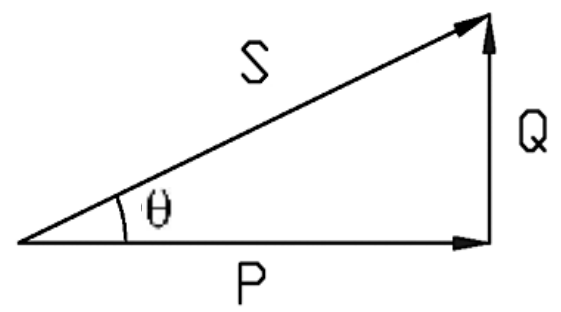

Figura 2 - Relação entre potências aparente (S), ativa $(P)$ e reativa $(Q)$.

\section{Metodologia}

O trabalho se classifica, segundo Gil (2000), como uma pesquisa do tipo estudo de caso, pois foi iniciado pelo planejamento com a formulação do problema e pela seleção da unidade-caso, no qual foi definido o número de casos. Após a fase de planejamento, ocorreu a fase da coleta de dados, e em seguida a sua análise e avaliação. Finalizada esta análise, apresentou-se os resultados obtidos.

Os procedimentos metodológicos foram iniciados por uma revisão bibliográfica, que teve por finalidade nivelar o conhecimento dos pesquisadores sobre o tema, além de obter dados para a discussão dos resultados.

A área de estudo a ser utilizada na coleta dos dados foi definida com a própria residência de um dos pesquisadores. O local serviu para se medir o consumo e a quantidade de potência reativa consumida, e assim levantar todos os dados para permitir os cálculos e dimensionamento dos capacitores.

Com os dados coletados, foi possível desenvolver um estudo que teve como ênfase a proposição de uma correção do fator de potência nos consumidores de pequeno porte.

\subsection{Caracterização da Área de Estudo}

A área de estudo consiste em um apartamento, composto de oito (08) cômodos, possuindo uma área construída de $110 \mathrm{~m}^{2}$, localizado na Rua dos Bandolins, Conjunto Califórnia I, Belo Horizonte, Minas Gerais, onde residem quatro (04) pessoas.

\section{Resultados e Discussão}

\section{1 ÁreA DE Estudo}

A área estudada está localizada na Regional Noroeste, conforme divisão das regiões de Belo Horizonte (Figura 3). Nesta região predominam residências com medidor eletromecânico monofásico $127 \mathrm{~V} / 120 \mathrm{~A}$, semelhantes ao do caso em estudo. 


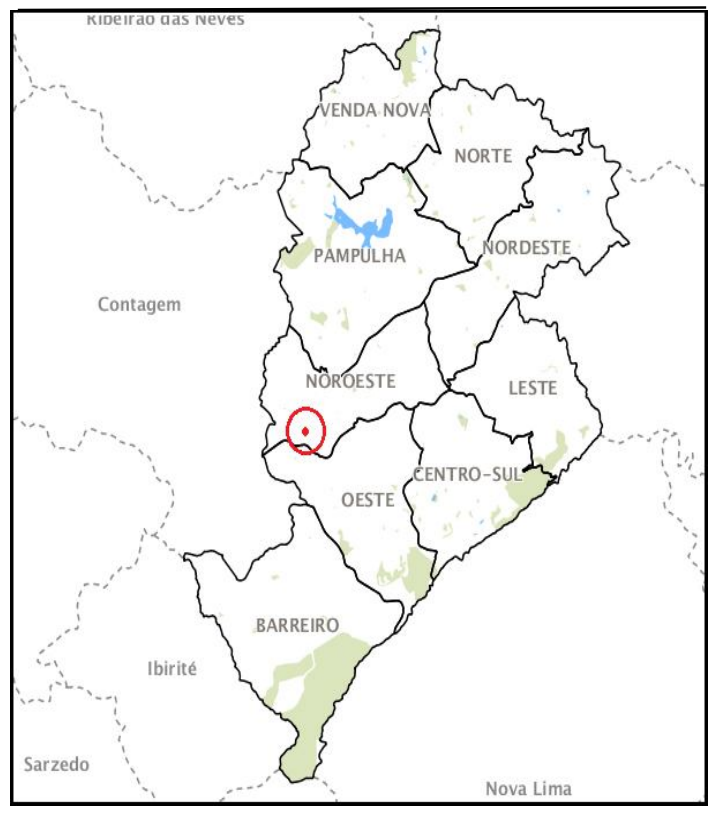

Figura 3 - Mapa de localização do ponto estudado na Região noroeste, conforme divisão do município de Belo Horizonte, MG.

Fonte: PBH, 2013, adaptado.

\subsection{Dados Coletados}

Para estudar como se comporta o fator de potência de uma instalação elétrica residencial ao longo de um dia, foi necessário obter valores das potências ativas dos equipamentos eletroeletrônicos de uso residencial, bem como seus fatores de potência fornecidos pelos fabricantes. A medição foi realizada utilizando um Medidor de Potência Fluke modelo 41B, obtendo assim os valores de potências ativas, reativas e aparentes da instalação em intervalos de 15 minutos.

Através desses dados, foi calculado o fator de potência da instalação para cada intervalo de tempo.
O Gráfico 1 apresenta o comportamento do fator de potência medido durante os intervalos, o fator de potência médio $(0,78)$ e o fator de potência ponderado (levando em conta a carga), 0,83.

O Gráfico 1 permitiu aferir que Fator de Potência sofre uma grande variação durante o período, dependendo da natureza das cargas utilizadas.

O Gráfico 2 apresenta um comparativo entre as energias ativa e reativa consumidas durante um dia. Neste é possível observar que ocorrem picos de consumo de energia por volta de 6:00 e 19:00 horas (horário de ponta), fato este que coincide com os horários de utilização de equipamentos de grande consumo de energia, como chuveiros, fornos, entre outros, devido à preparação de saída e chegada dos consumidores nas residências.

Como a medição foi realizada em intervalos de 15 minutos, os dados foram concentrados para a obtenção de resultados de hora em hora.

O quadro das cargas instaladas, sem fator de demanda, observa-se na área de estudo. Nela podese reparar na diversidade dos fatores de potência das cargas, com ênfase no fator das lâmpadas fluorescentes compactas $(0,55)$.

As lâmpadas fluorescentes compactas foram indicadas como uma das soluções para a redução do consumo de energia em uma residência, mas, devido ao seu baixíssimo fator de potência, estas consomem quase o dobro da energia, ou seja, uma lâmpada de $26 \mathrm{~W}$ consome 47,27 VA. 


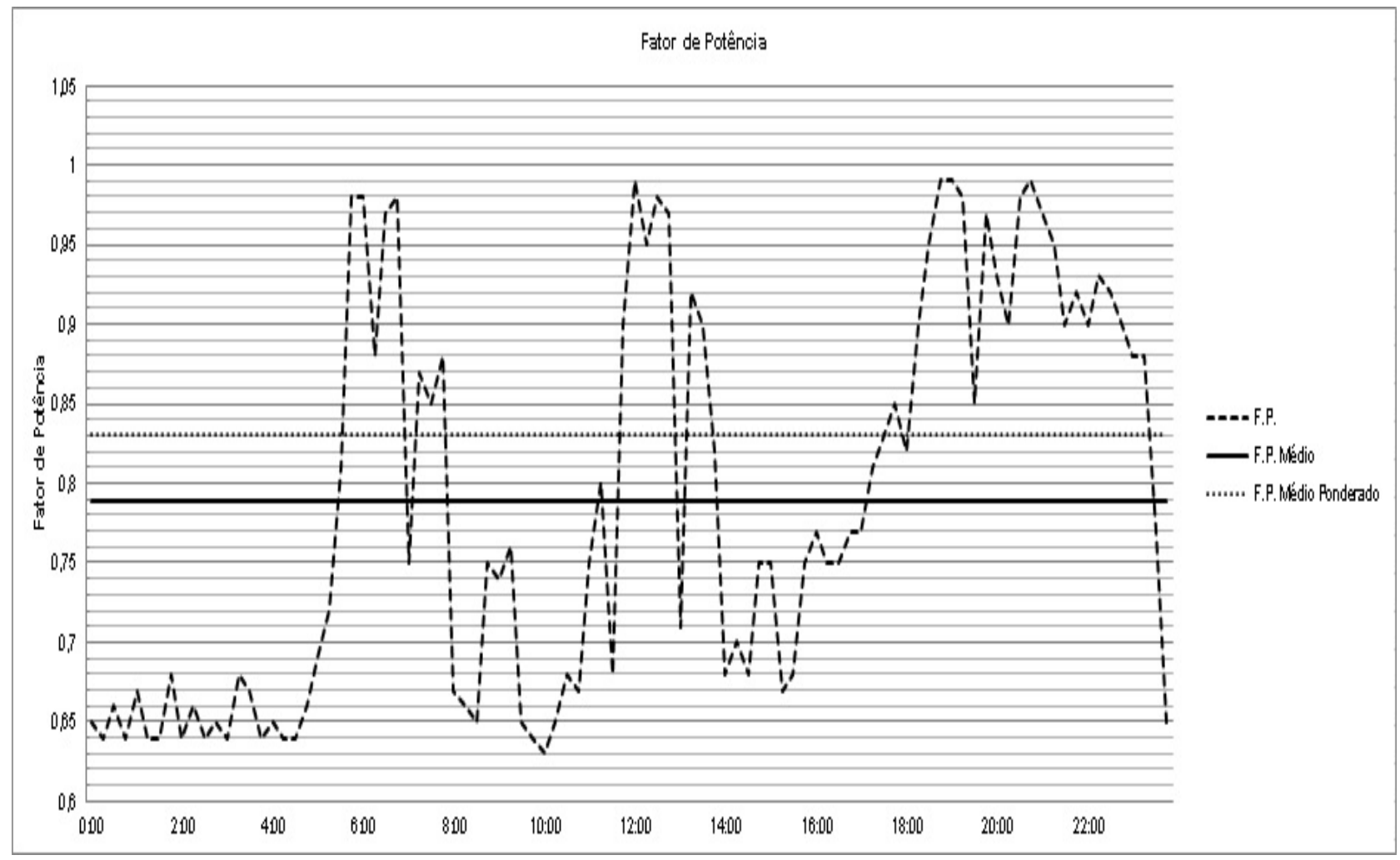

Gráfico 1 - Medição de Fator de Potência

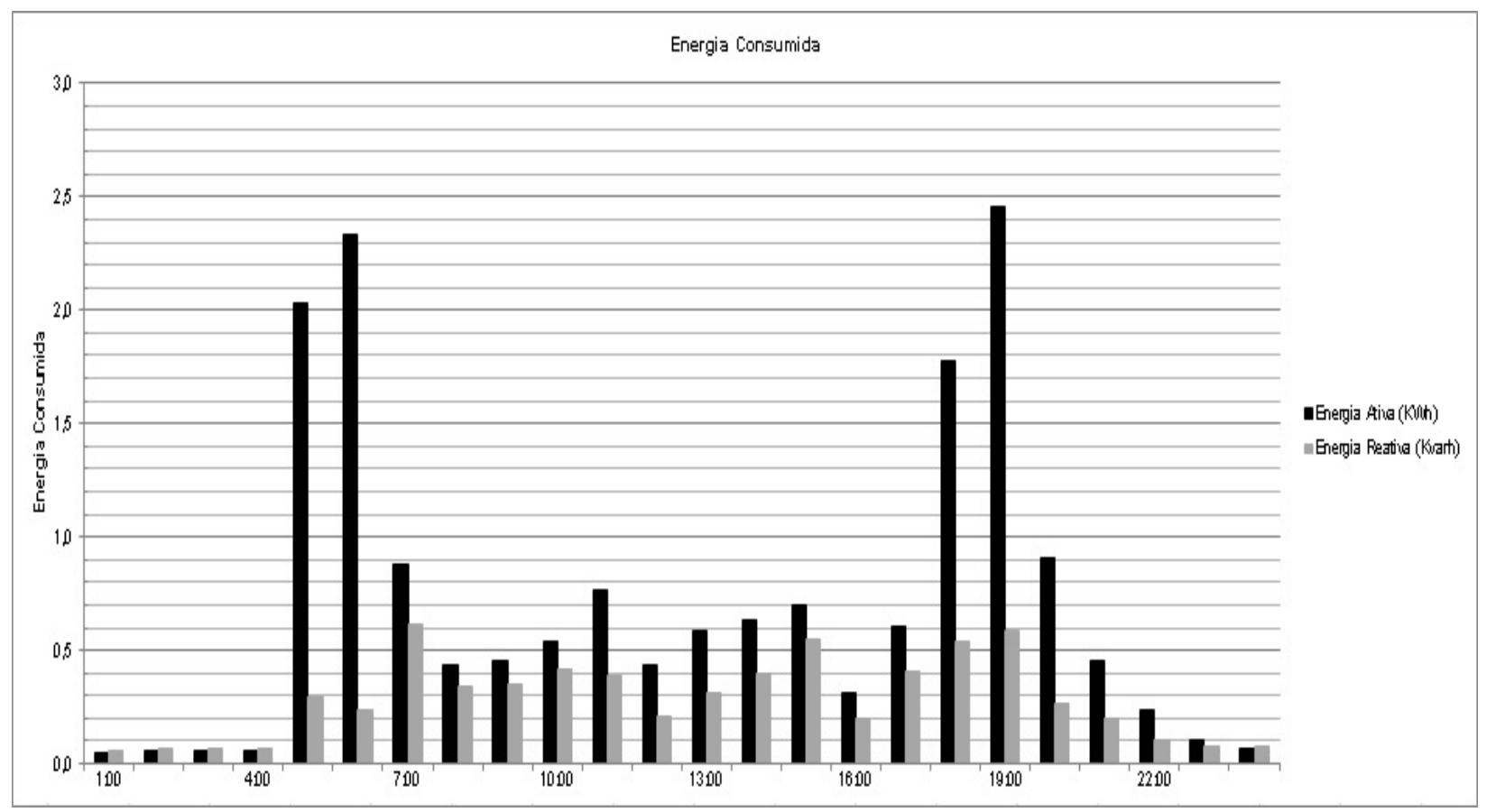

Gráfico 2 - Medição da Energia Consumida 


\subsection{CÁlculo de Potência Reativa}

\subsubsection{Fator de Potência MÉdio Ponderado}

O fator de potência médio ponderado foi calculado através do somatório das multiplicações entre os fatores de potência e as cargas na hora da medição, divididas pelo somatório das cargas medidas, conforme demonstrado na Equação 1.

$$
\begin{gathered}
F P(\text { ponderado })=\Sigma(P(w) * F P) / \Sigma(P(w))[\text { Eq. 1] } \\
F P(\text { ponderado })=\frac{14057,7}{16920} \\
F P(\text { ponderado })=0.8303
\end{gathered}
$$

Este fator de potência ponderado foi assim calculado devido à variabilidade do consumo de energia em uma instalação elétrica residencial.

\subsubsection{POTÊnCIA REATIVA dO BANCO DE}

\section{Capacitores}

Procedeu-se ao cálculo da potência reativa necessária utilizando-se os dados fornecidos pelo banco de capacitores. Através do resultado obtido, o fator de potência foi elevado até os patamares exigidos pela legislação vigente.

A potência reativa do capacitor foi calculada pela Equação 2 proposta por Mamede Filho (2007, p. 214).

$$
Q(\operatorname{var})=P(w) *(\operatorname{TAN}(\operatorname{ARCOS}(F P 1))-\operatorname{TAN}(\operatorname{ARCOS}(\mathrm{FP} 2)))
$$

$$
\text { [Eq. 2] }
$$

Onde:

FP1 = Fator de potência atual da instalação; FP2 = Fator de potência desejado.

Calculando-se:

$$
\begin{gathered}
Q(\text { var })=845,90 *(\operatorname{TAN}(\operatorname{ARCOS}(0,83))-\operatorname{TAN}(\operatorname{ARCOS}(0,92))] \\
Q(\mathrm{var})=845,90 *(0,67-0,42)
\end{gathered}
$$

$$
Q=208,09 \text { Var }
$$

\subsubsection{VALOR DA CAPACITÂNCIA DO CAPACITOR}

O valor da capacitância do capacitor foi calculada pela Equação 3, proposta por Weg (2009, p. 12).

$$
C\left(\mu F^{\prime}\right)=\underset{24 \pi+f+w^{2}}{Q[\operatorname{var}}[\text { [Eq. 3] }
$$

onde:

$$
\begin{aligned}
& Q(\text { Var })=\text { Potência reativa necessária; } \\
& f=\text { Frequência da rede; } \\
& V=\text { Tensão de operação. }
\end{aligned}
$$

Calculando-se:

$$
\begin{aligned}
& C(\mu F)=\frac{208,09}{377+127^{2}} \\
& C(\mu F)=\frac{208,09}{6080633} \\
& C(\mu F)=34,22 \mu F
\end{aligned}
$$

\subsection{ANÁlise do RESULTADo FinAL}

Após o levantamento dos dados e a realização dos cálculos, pode-se perceber que a economia gerada com a correção do fator de potência é bastante significativa quando se leva em consideração todas as residências existentes no Brasil.

O setor residencial brasileiro consumiu 114.797 GWh de Energia Elétrica no período de junho de 2011 a junho de 2012 e possui atualmente 60.724 .000 de consumidores residenciais registrados no cadastro das concessionárias de distribuição. A média de consumo de energia por ligação residencial foi de $157,5 \mathrm{kWh} / \mathrm{mês}$ (EPE, 2012). Descontando-se os fatores horo-sazonais, pode-se definir que cada 
residência do país possui uma carga de 215,75 Watts ligada constantemente na rede.

O investimento para correção do fator de potência nas residências ainda é dispendioso, mas o retorno do investimento ocorre por volta de 5 meses após a instalação.

Por tratar-se de um produto importado, um pequeno controlador de fator de potência residencial com um banco de capacitores composto de um capacitor de 20uF (Figura 4), dois de 10uF e um $5 \mu \mathrm{F}$ tem um custo médio no mercado de $\mathrm{R} \$ 170,00$.

Trata-se de uma tecnologia bastante conhecida e, com a produção de similares nacionais, a tendência deste preço é cair drasticamente.

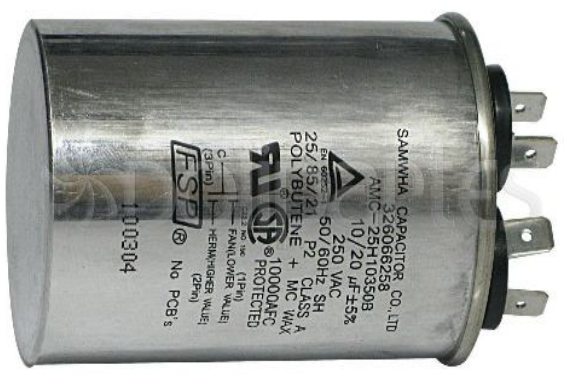

Figura 4 - Capacitor de $20 \mu \mathrm{F}$. Fonte: Samwa Capacitores

O apêndice 1 é caracterizado pela planilha de levantamento de cargas que após tratadas geraram a Tabela 2, que contém os resultados dos dados levantados na Residência Pesquisada, destacando a comparação dos resultados obtidos com e sem a correção do fator de potência.
Tabela 2 - Cálculo de potência ativa, reativa, fator de potência e capacitor.

\begin{tabular}{|l|r|}
\hline \multicolumn{2}{|c|}{ Dados Consolidados } \\
\hline Carga Instalada (W) & 8459,00 \\
\hline Fator de Demanda & 0,10 \\
\hline Carga Demandada (W) & 845,90 \\
\hline $\begin{array}{l}\text { Fator de Potência médio ponderado da } \\
\text { Instalação }\end{array}$ & 0,83 \\
\hline Fator de Potência Desejável & 0,92 \\
\hline \multicolumn{2}{|c|}{ Dados sem correção de fator de potência } \\
\hline Potência Reativa (var) & 568,45 \\
\hline Potência Aparente (VA) & 1019,15 \\
\hline Corrente (A) & 8,02 \\
\hline \multicolumn{2}{|c|}{ Dados com correção de fator de potência } \\
\hline $\begin{array}{l}\text { Potência do Capacitor para correção } \\
\text { do FP (var) }\end{array}$ & 208,09 \\
\hline Valor do Capacitor ( $\mu$ F) & 34,22 \\
\hline Potência Reativa (var) & 360,35 \\
\hline Potência Aparente (VA) & 919,45 \\
\hline Corrente (A) & 7,23 \\
\hline Percentual de redução da corrente & $\mathbf{9 , 8 5 \%}$ \\
\hline
\end{tabular}

\section{CONCLUSÃo}

O fator de potência de uma instalação elétrica residencial apresentou uma variação intensa e contínua ao longo das 24 horas do dia, em oposição às instalações elétricas industriais ou comerciais, em que é possível obter um padrão bem definido. Portanto, um mecanismo de correção em uma residência precisaria ter a capacidade de acompanhar esta variabilidade, como é o caso dos bancos automáticos de capacitores através dos controladores de fator de potência.

De acordo com a Resolução no 414 da ANEEL (Agência Nacional de Energia Elétrica), estabelece-se o fator de potência mínimo de 0,92 para instalações Classe A e Classe B, com cobrança sobre excedente reativo. 
Assim, quando tal resolução for estendida ao setor residencial, como já previsto, haverá horários em que o fator de potência será muito menor que o estabelecido, sendo igualmente taxado pelo excedente de energia reativa. Acredita-se que, em um futuro próximo, com a constante procura por uma maior eficiência energética, a taxação do consumo de energia reativa no setor residencial será uma realidade.

Considerando que, com a correção do fator de potência, a corrente circulante em cada residência fosse reduzida na mesma proporção do caso estudado em $9,85 \%$, em um universo de 60,7 milhões de ligações residenciais, seria obtida uma redução bastante expressiva das perdas joulicas no sistema.

Por estas serem proporcionais ao quadrado da corrente circulante, isto beneficiaria de forma significativa o sistema de geração e transmissão de energia, que trabalharia com maior folga. Tal fato melhora a qualidade da energia elétrica, favorece a otimização do transporte da energia e diminui a necessidade de investimentos a curto e médio prazo.

Além disso, haveria menor utilização das usinas termoelétricas de distribuição, o que traz como principais vantagens a menor poluição do meio ambiente e a redução de gastos para os cofres públicos, e, indiretamente, para a economia brasileira. Todos estes fatores justificam a correção do fator de potência também das cargas residenciais.

\section{AGRADECIMENTOS}

À CEMIG, por disponibilizar o material para pesquisa $\mathrm{e}$ o compartilhamento de algumas informações. Também aos professores do UniBH que ajudaram a desenvolver a pesquisa e a toda comunidade científica pelo compartilhamento de informações que contribuiu para o enriquecimento das discussões dos resultados.

\section{REFERÊNCIAS}

ANEEL - AGÊNCIA NACIONAL DE ENERGIA ELÉTRICA. RESOLUÇÃO ANEEL № 414, DE 9 DE SETEMBRO DE 2010. Estabelece, de forma atualizada e consolidada, as Condições Gerais de Fornecimento de Energia Elétrica. Brasília/DF: ANEEL, $2010 . \quad$ Disponível em < http://www.aneel.gov.br/cedoc/ren2010414comp.pdf>. Acesso em: 18 set. 2012.

CEMIG - Companhia Energética de Minas Gerais Manual de Instalações Elétricas Residenciais. Belo Horizonte - MG: CEMIG, 2003. 218 p.

CREDER, Hélio. Instalações Elétricas. 15aㅡ ed. Rio de Janeiro/RJ: LTC, 2007. 428 p.

EPE - Empresa de Pesquisa Energética - Boletim de Conjuntura Energética - 2을 trimestre de 2012. Brasília/DF: EPE, 2012. 83p. Disponível em <http://www.epe.gov.br. Acesso em: 05 abr. 2013.
GIL, A. C. Como elaborar projetos de pesquisa. 4. ed. São Paulo: Atlas, 2000. 176 p.

MAMEDE FILHO, João. Instalações Elétricas Industriais. $7^{\mathfrak{a}}$ ed. São Paulo/SP: LTC, 2007. 908 p.

PBH - PREFEITURA DE BELO HORIZONTE. Gestão Compartilhada. Belo Horizonte/MG: PBH, 2013. Disponível em $<$ http://gestaocompartilhada.pbh.gov.br/>. Acesso em: 22 abr. 2013.

WEG Automação S.A. - Manual para Correção do Fator de Potência. Jaraguá do Sul/SC: WEG, 2009. 40 p. Disponível em: <http://www.weg.net>. Acesso em: 28 out. 2012. 


\begin{tabular}{|c|c|c|c|c|c|c|c|c|}
\hline \multicolumn{9}{|c|}{ Quadro de cargas - Residência 1} \\
\hline Cômodos & Equipamentos & Quantidade & $\begin{array}{c}\text { Tensão } \\
\text { (V) }\end{array}$ & Potência (W) & F.P. & $\begin{array}{l}\text { Potência } \\
\text { Ativa (W) }\end{array}$ & $\begin{array}{c}\text { Potência } \\
\text { Aparente } \\
\text { (VA) }\end{array}$ & $\begin{array}{c}\text { Potência } \\
\text { Reativa (Var) }\end{array}$ \\
\hline \multirow{2}{*}{ Sala } & Lâmpada Fluorescente compacta & 5 & 127 & 26 & 0,55 & 130,00 & 236,36 & 197,40 \\
\hline & Televisão de LCD - 42" & 1 & 127 & 150 & 0,85 & 150,00 & 176,47 & 92,96 \\
\hline \multirow{3}{*}{ Quarto 1} & Televisão de Tubo - 14" & 1 & 127 & 46 & 0,85 & 46,00 & 54,12 & 28,51 \\
\hline & Computador & 1 & 127 & 150 & 0,80 & 150,00 & 187,50 & 112,50 \\
\hline & Lâmpada Fluorescente compacta & 2 & 127 & 26 & 0,55 & 52,00 & 94,55 & 78,96 \\
\hline \multirow{3}{*}{ Quarto 2} & Televisão de tubo - 14" & 1 & 127 & 150 & 0,80 & 150,00 & 187,50 & 112,50 \\
\hline & Computador & 1 & 127 & 150 & 0,80 & 150,00 & 187,50 & 112,50 \\
\hline & Lâmpada Fluorescente compacta & 2 & 127 & 26 & 0,55 & 52,00 & 94,55 & 78,96 \\
\hline \multirow{2}{*}{ Quarto 3} & Televisão de Led 32" & 1 & 127 & 135 & 0,85 & 135,00 & 158,82 & 83,67 \\
\hline & Lâmpada Fluorescente compacta & 1 & 127 & 26 & 0,55 & 26,00 & 47,27 & 39,48 \\
\hline \multirow{2}{*}{ Banheiro } & Chuveiro & 1 & 127 & 4400 & 1,00 & 4400,00 & 4400,00 & 0,00 \\
\hline & Lâmpada Fluorescente compacta & 3 & 127 & 26 & 0,55 & 78,00 & 141,82 & 118,44 \\
\hline \multirow{4}{*}{$\begin{array}{l}\text { Área de } \\
\text { Serviço }\end{array}$} & Máquina de Lavar & 1 & 127 & 1000 & 0,60 & 1000,00 & 1666,67 & 1333,33 \\
\hline & Secadora de Roupa & 1 & 127 & 1000 & 0,80 & 1000,00 & 1250,00 & 750,00 \\
\hline & Máquina de Costura & 1 & 127 & 300 & 0,60 & 300,00 & 500,00 & 400,00 \\
\hline & Lâmpada Fluorescente compacta & 1 & 127 & 26 & 0,55 & 26,00 & 47,27 & 39,48 \\
\hline \multirow{3}{*}{ Cozinha } & Geladeira & 1 & 127 & 150 & 0,98 & 150,00 & 153,06 & 30,46 \\
\hline & Micro-ondas & 1 & 127 & 400 & 0,60 & 400,00 & 666,67 & 533,33 \\
\hline & Lâmpada Fluorescente compacta & 2 & 127 & 32 & 0,55 & 64,00 & 116,36 & 97,18 \\
\hline
\end{tabular}

